\title{
\begin{tabular}{l|l} 
Mitraries & DSpace@MIT
\end{tabular}
}

\author{
MIT Open Access Articles
}

Optical design for a spatial-spectral
volume holographic imaging system

The MIT Faculty has made this article openly available. Please share how this access benefits you. Your story matters.

Citation: Gelsinger-Austin, Paul J., Yuan Luo, Jonathan M. Watson, Raymond K. Kostuk, George Barbastathis, Jennifer K. Barton and Jose M. Castro, "Optical design for a spatial-spectral volume holographic imaging system", Opt. Eng. 49, 043001 (Apr 12, 2010); (c) 2010 SPIE.

As Published: http://dx.doi.org/10.1117/1.3378025

Publisher: Society of Photo-optical Instrumentation Engineers

Persistent URL: http://hdl.handle.net/1721.1/61418

Version: Final published version: final published article, as it appeared in a journal, conference proceedings, or other formally published context

Terms of Use: Article is made available in accordance with the publisher's policy and may be subject to US copyright law. Please refer to the publisher's site for terms of use. 


\section{Optical design for a spatial-spectral volume holographic imaging system}

Paul J. Gelsinger-Austin

The University of Arizona

College of Optical Sciences

Tucson, Arizona 85721

Yuan Luo, MEMBER SPIE

Jonathan M. Watson

Massachusetts Institute of Technology

Department of Mechanical Engineering

Cambridge, Massachusetts 02139

\section{Raymond K. Kostuk}

The University of Arizona

College of Optical Sciences and

Department of Electrical and Computer Engineering

Tucson, Arizona 85721

\section{George Barbastathis}

Massachusetts Institute of Technology

Department of Mechanical Engineering

Cambridge, Massachusetts 02139

Jennifer K. Barton, MEMBER SPIE

The University of Arizona

College of Optical Sciences

and

Department of Electrical and Computer Engineering

and

Division of Biomedical Engineering

Tucson, Arizona 85721

Jose M. Castro

The University of Arizona

Department of Electrical and Computer Engineering

Tucson, Arizona 85721

jmcastro0117@gmail.com

\begin{abstract}
The spatial-spectral holographic imaging system $\left(\mathrm{S}^{2}-\mathrm{VHIS}\right)$ is a promising alternative to confocal microscopy due to its capabilities to simultaneously image several sample depths with high resolution. However, the field of view of previously presented $S^{2}$-VHIS prototypes has been restricted to less than $200 \mu \mathrm{m}$. We present experimental results of an improved $S^{2}$-VHIS design that has a field of view of $\sim 1 \mathrm{~mm}$ while maintaining high resolution and dynamic range. () 2010 Society of PhotoOptical Instrumentation Engineers. [DOI: 10.1117/1.3378025]
\end{abstract}

Subject terms: medical imaging; holography applications; optical system design; microscopes; confocal optics; holographic optical elements.

Paper 090919R received Nov. 25, 2009; revised manuscript received Feb. 13 , 2010; accepted for publication Feb. 16, 2010; published online Apr. 12, 2010.

\section{Introduction}

Spectroscopic and microscopic imaging instruments are essential tools in biological and medical sciences. Instruments that combine both techniques into the same instrument can further enhance the understanding of biological processes. Sophisticated systems such as combined optical coherence tomography/laser induced fluorescence, ${ }^{1}$ multispectral confocal microendoscopy, ${ }^{2}$ and computed tomographic imaging spectroscopy ${ }^{3}$ provide the capability of collecting both

0091-3286/2010/\$25.00 @ 2010 SPIE spatial and spectral data about an object simultaneously. However, each requires the use of mechanical scanning to build up an entire 4-D $(x, y, z, \lambda)$ dataset that represents a physical object.

A spectral-spatial volume holographic imaging system $\left(\mathrm{S}^{2}\right.$-VHIS $)$ is an optical device that minimizes the need for mechanical scanning. Therefore, for an given volume, images can be obtained faster using $S^{2}$-VHIS than using confocal microscopes or optical coherent tomography systems (OCTs). The limitations of $\mathrm{S}^{2}$-VHIS and confocal microscopes are the depth penetrations, which are limited to $<200 \mu \mathrm{m}$ compared with $<1 \mathrm{~mm}$ of OCT systems. ${ }^{4}$ In 


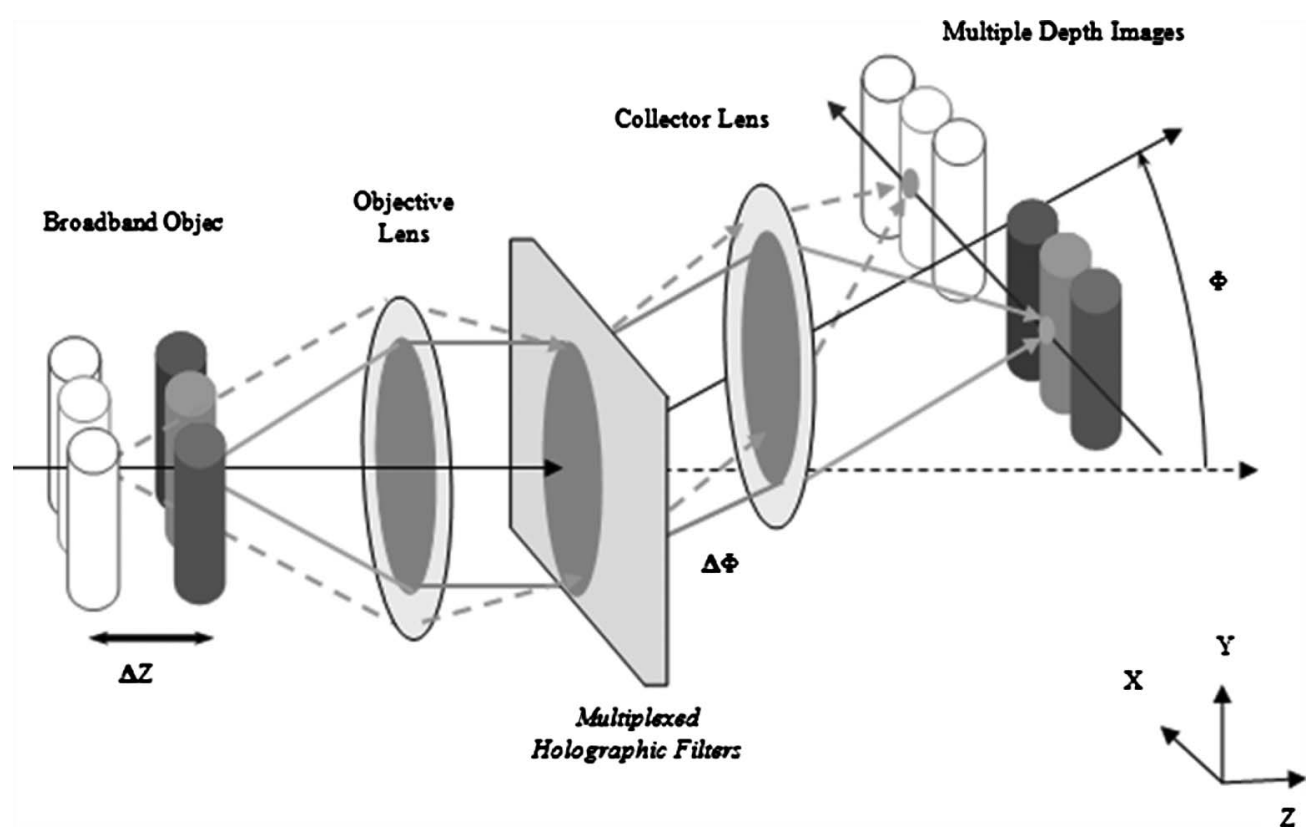

Fig. 1 Spatial-spectral volume holographic imaging system.

terms of lateral resolution, $S^{2}$-VHIS can theoretically achieve similar resolution than confocal microscopes. In practice, this requires a lot of improvements in the spectrum-angular selectivity of the hologram. $\mathrm{S}^{2}$-VHIS depth resolution is currently comparable with OCT systems.

The key to this device is a volume holographic element that converts a 4-D distribution of information to a 2-D distribution. The 4-D distribution being converted is, in general, a collection of point scattering elements with dimensions $(x, y, z)$ and band-limited emission spectra with mean wavelength $\lambda_{0}$ and bandwidth $\Delta \lambda$.

The functionalities of the $\mathrm{S}^{2}$-VHIS system have been previously described. ${ }^{4-7}$ Conceptual designs have been implemented in $\mathrm{LiNbO}^{5,6}$ and more recently in doped polymer, ${ }^{7}$ achieving excellent optical sectioning capabilities in a field of view $<200 \mu \mathrm{m}$.

In this work, we present an improved $\mathrm{S}^{2}$-VHIS design that enables the observation of a field several times larger while maintaining micron-scale resolution.

The remainder of the work consists of five sections. In Sec. 2 we summarize the operation of $S^{2}$-VHIS. Section 3 details the system requirements to improve the field of view. The design of the components is described in Sec. 4. Experimental results are shown in Sec. 5. Section 6 summarizes our results.

\section{Basic Description of Spatial-Spectral Holographic Imaging System}

As shown in Fig. 1, $S^{2}$-VHIS consists of a 4-f configuration of lenses with a holographic element placed in the Fourier plane. The holographic element is composed of anglemultiplexed planar and spherical wave gratings. The nominal gratings for this device are optimized for infinite conjugates, where a spherical (or plane) wave incident on the hologram generates a diffracted plane wave. The holographic element is considered a thick phase grating, and can be analyzed exactly using coupled wave theory ${ }^{7-9}$ or Fourier Optics in the weak diffraction approximation. ${ }^{10}$

The diffraction efficiency of the hologram is very sensitive to the wavelength and shape of the incident wavefront. Due to this feature, the hologram acts as a wavefront filter. The action of the filter is to select only those wavefronts that originate at a particular depth from within the scattering object, which are within the designed spectral band. The optical section width (axial or $z$ resolution) is determined by how well the hologram is able to reject wavefronts generated by out-of-plane point sources. An analytical solution quantifying the optical sectioning performance of this system has been derived for simple planar grating structures. ${ }^{5,6}$ More complicated structures require the use of numerical analysis. ${ }^{9,10}$ For the purposes of designing the system architecture, all that is necessary for simulation of the hologram is the wavelength-angle relationship defined by the Bragg condition of a planar grating. ${ }^{9}$ This condition gives the correct angle of incidence $(\theta)$ of the on-axis beam for a given wavelength. The optical axis of the system is rotated, bent by twice the angle of incidence, giving the equation $\Phi(\lambda)=2 \theta(\lambda)$. Thus, the wavelength and $x$ axes are nonorthogonal, but they may be decoupled by using multiplexed gratings with matched responses to specific wavelengths/wavefronts. Dispersion in the hologram ultimately limits the spatial resolution along the $x$ axis as well as the spectral resolution. The lack of grating periodicity along the $y$ axis results in a degeneracy that provides the $y$ axis field of view. In the diffraction limit, the resolution along this axis is limited by the numerical aperture of the objective lens.

Multiplexing many gratings into the same volume allows for the observation of multiple planes in the object simultaneously. ${ }^{5}$ Given properly designed multiplexed gratings, it is possible to map any point $(x, y, z)$ in object space to a point $(x, y)$ in image space. The holographic sectioning 
(a)

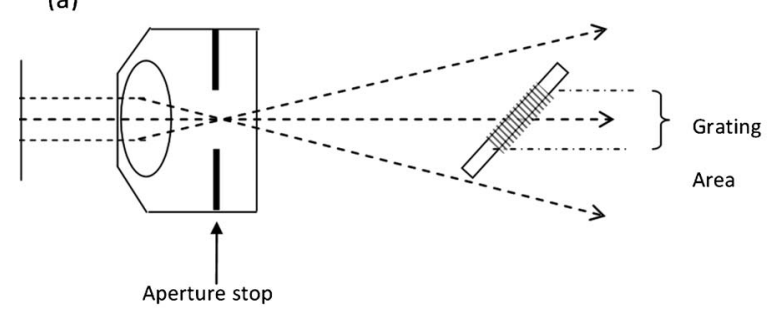

(b)

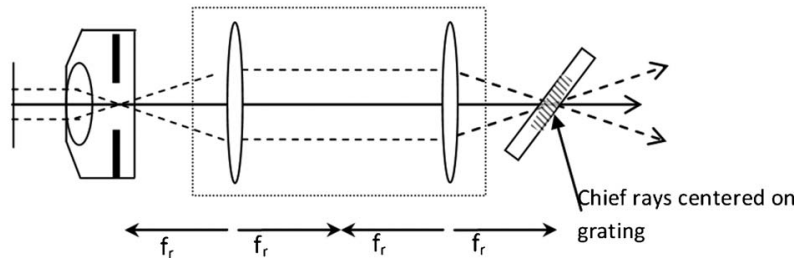

Fig. 2 (a) Layout showing vignetted chief rays, and (b) layout employing a relay system.

capability of the $S^{2}$-VHIS bears some resemblance to the action of a slit in confocal microscopy ${ }^{11}$ with two differences: 1 . the multiplexing property of volume holograms allows sections from multiple "slits" to be viewed simultaneously, and 2. the dispersion property of volume gratings allows the instrument to also have spectral sectioning capability. Also, there are no special illumination conditions for this system other than that the object must be incoherently radiating light with some appreciable bandwidth. This means that reflection and transmission with spatially incoherent illumination and fluorescence modes of operation are valid for this instrument.

\section{Optical Design Requirements}

We designed an improved $\mathrm{S}^{2}$-VHIS that enables the observation of a field several times larger than previously realized, while maintaining micron-scale resolution. According to Ref. 5, the system performs well when using objective and collector lenses corrected for infinite conjugates. This is because near-collimated light reduces aberrations in the diffracted beam produced by the hologram. As this system is intended for use in microscopy, the system was designed to be telecentric in object space to maintain constant lateral magnification with axial location in the sample. For this system to be telecentric in object space and unvignetted over an appreciable field of view, the hologram must be the limiting aperture in the system, otherwise known as the aperture stop.

The previous system, as illustrated in Fig. 1, used commercially available long-working-distance microscope objectives and suffered from vignetting. A common feature of lenses of this type is that that the aperture stop is at an inaccessible location inside the objective housing. Therefore, it is not feasible to place the hologram with the pupil without auxiliary optics. This results in vignetting, as shown in Fig. 2(a). The remedy to this problem is to insert

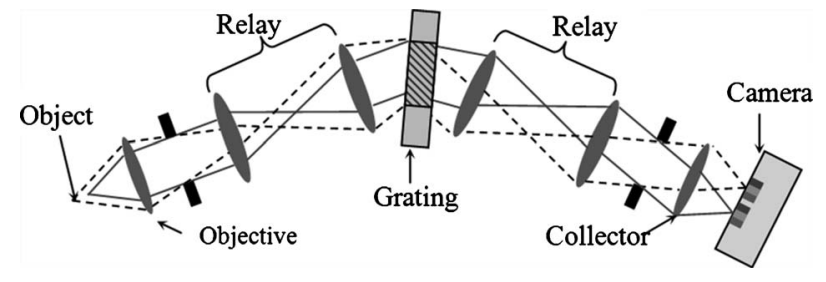

Fig. 3 Paraxial design of the S2-VHIS with relays.

a telecentric relay into the system, such that the aperture stop of the objective lens is imaged onto the hologram, as shown in Fig. 2(b).

\section{Component Level Design}

The resolutions along the lateral and axial dimensions are primarily determined by the numerical aperture of the objective lens and the thickness of the holographic grating, respectively. It was experimentally determined that an objective lens numerical aperture of $0.55(\mathrm{fo}=3.6 \mathrm{~mm})$ and a grating thickness of $1.8 \mathrm{~mm}$ leads to an axial resolution of $22 \mu \mathrm{m}$ and lateral resolution of $4.5 \mu \mathrm{m}$ for a wavelength of $633 \mathrm{~nm}$. Increasing the objective NA or hologram thickness can reduce the section thickness further. ${ }^{5,6}$ The collector lens' focal length (fc) is chosen based on the focal length of the objective lens and the desired lateral magnification |fc/ fol. A Lumenera (Ottawa, Canada) 6-Mpixel chargecoupled device (CCD) array with a pixel pitch of $3.5 \mu \mathrm{m}$ is used for imaging in reflectance mode, and a highly sensitive Andor (Belfast, Ireland) iXon array with a pixel pitch of $16 \mu \mathrm{m}$ is used for fluorescence mode imaging. In combination with these cameras, collector focal lengths of $10 \mathrm{~mm}$ (Lumenera) and $20 \mathrm{~mm}$ (Andor) are used to resolve lateral features smaller than $15 \mu \mathrm{m}$. Both objective and collector lenses are achromatic, because the system functions over a $100-\mathrm{nm}$ band in the visible wavelength range of 486 to $656 \mathrm{~nm}$. The lenses used are commercially available microscope objectives with long working distances. The objective lens is an Olympus ULWDMSPlan 50× (NA $=0.55, \mathrm{f}=3.6 \mathrm{~mm})$, and the collector lenses are Mitutoyo (Aurora, Illinois) M Plan APO $20 \times \quad(\mathrm{NA}=0.42$, f $=10.0 \mathrm{~mm})$ and $M$ Plan APO $10 \times \quad(\mathrm{NA}=0.28$, $\mathrm{f}$ $=20.0 \mathrm{~mm}$ ). See Figs. 3 and 4 .

The form of the telecentric relay system can be approached from first-order paraxial optics, and basic thirdorder aberration analysis. A focal length of $33 \mathrm{~mm}$ is used for each element, as this focal length allows sufficient working distance at both ends of the relay while using standard 1-in. diam optics. The wavefront error tolerance for the relay is derived from the optical section thickness $\Delta z$. The maximum allowed wavefront distortion that can be tolerated by the hologram can be approximated by defocus, and is given by $\Delta W_{\text {defocus }}= \pm 1 / 2(\Delta z / 2)(\mathrm{NA})^{2}{ }^{2}{ }^{11}$ For a 0.55 -NA objective at a wavelength of $633 \mathrm{~nm}$, this corre-

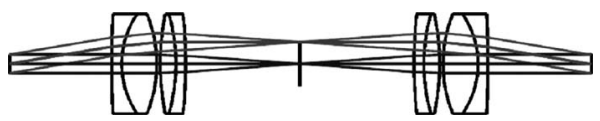

Fig. 4 Layout of the optical relay system. 
sponds to a wavefront change of approximately $2.6 \lambda$ for $\Delta z \sim 22 \mu \mathrm{m}$. Therefore, an OPD change of $2.6 \lambda$ between the on-axis and full-field object points is all that is allowable to achieve high contrast optical slicing over the field of view.

Since the design is symmetrical, odd aberrations are cancelled and only even aberrations need be considered. Of the even aberrations, field curvature is of less importance, since modest curvature in the object plane is acceptable. The aberrations of main concern are therefore spherical aberration, astigmatism, and axial chromatic aberration. Achromatic doublets were chosen for our design to correct for spherical and chromatic aberrations. ${ }^{12}$ The relay as designed uses a combination of catalog achromatic doublets, and can be thought of as two back-to-back Ploessl-type eyepieces, ${ }^{13}$ where each Ploessl system consists of two achromats facing each other. Aberrations are sufficiently optimized to provide a MTF contrast greater than 0.6 for a $15-\mu \mathrm{m}$ feature over a $1.2-\mathrm{mm}$ field of view, although the full unvignetted field of view is $\sim 2 \mathrm{~mm}$.

\section{Experimental Results}

A multiplexed hologram was formed by superimposing interferometric exposures in the same volume of phenathrenequinone (PQ)-doped poly(methyl methacrylate) (PMMA) photopolymer recording material. The material was recorded using an argon ion laser operating at a wavelength of $0.488 \mu \mathrm{m}$. A full-width half maximum (FWHM) angular bandwidth of $\sim 0.03 \mathrm{deg}$ was obtained for each grating. The FWHM spectral resolution was $0.2 \mathrm{~nm}$.

With the improved optical design, a well-corrected $y$ field of view of $1.2 \mathrm{~mm}$ has been achieved. Earlier experiments had established that without the addition of the relay systems, only about $125 \mu \mathrm{m}$ of the sample had been visible. The field of view in the $x$ dimension can be varied depending on the hologram design and the spectral bandwidth of the emitted light. In one realized system, it is $220 \mu \mathrm{m}$ with two multiplexed gratings and reflected light from an LED source with center wavelength of $633 \mathrm{~nm}$ and bandwidth of $25 \mathrm{~nm}$ (FWHM). The LED used was a Cree (Durham, North Carolina) XLamp XR7090RED. The Air Force Resolution Chart image in Fig. 5(a), taken in transmission mode, shows that lateral features as small as $4.5 \mu \mathrm{m}$ are well resolved by this system. The resolution along the $x$ axis is slightly worse than that along the $y$ axis due to dispersion in the hologram. A reflectance mode image taken using a human ovary sample shows two depth sections captured simultaneously, where the left-hand image is taken just below the surface of the ovary, and the right-hand image is designed to be approximately $70 \mu \mathrm{m}$ deeper in the tissue. Another realization of the instrument uses fluorescent stains as the dominant contributor to the object's spectral signal [Fig. 5(c)]. A sample of mouse colon was stained with acridine orange, then illuminated with a 355-nm wavelength laser. The emission spectrum of the fluorophore had an emission bandwidth of approximately $100 \mathrm{~nm}$ at a center wavelength of $\sim 550 \mathrm{~nm}$. This wide bandwidth increases the $x$ field of view to $0.5 \mathrm{~mm}$; thus the hologram was designed for greater angular separation of depth sections.

The depth sectioning capabilities of the improved $S^{2}$-VHIS design were also evaluated. A point source was

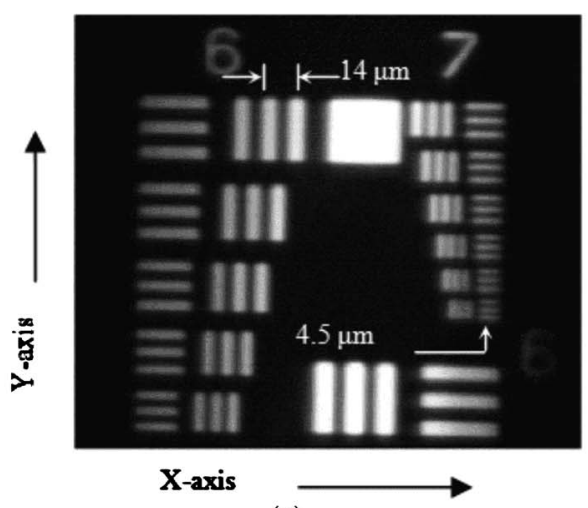

(a)

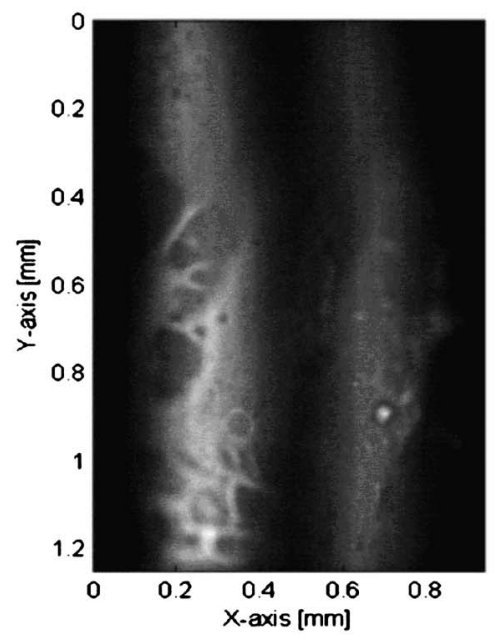

(b)

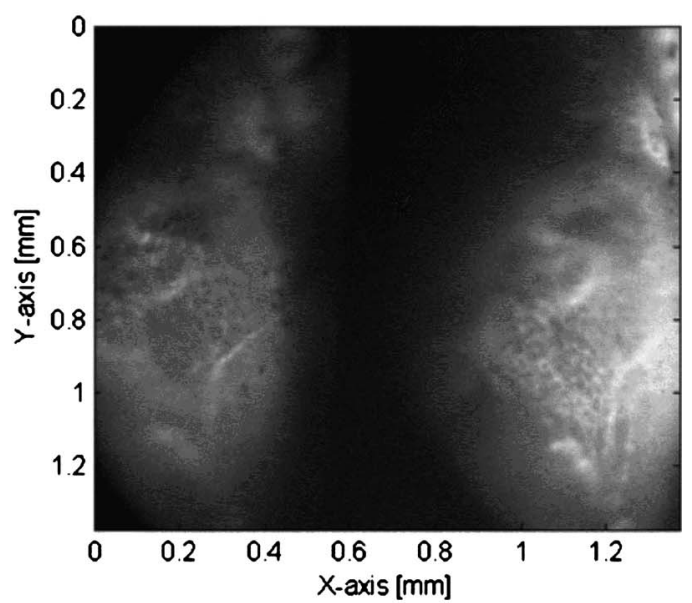

(c)

Fig. 5 Images taken with the S2VHIS: (a) detail of an image of a USAF bar target (transmission imaging), (b) human ovary (reflectance imaging), and (c) mouse colon (fluorescence imaging).

generated using a collimated 633-nm HeNe laser and a microscope objective placed on a motorized translation stage. Results shown in Fig. 6 indicate an FWHM axial resolution of $22.3 \mu \mathrm{m}$. Cross talk between depths separated $>70 \mu \mathrm{m}$ are $<10 \%$. This value can be further reduced by applying image processing techniques. 


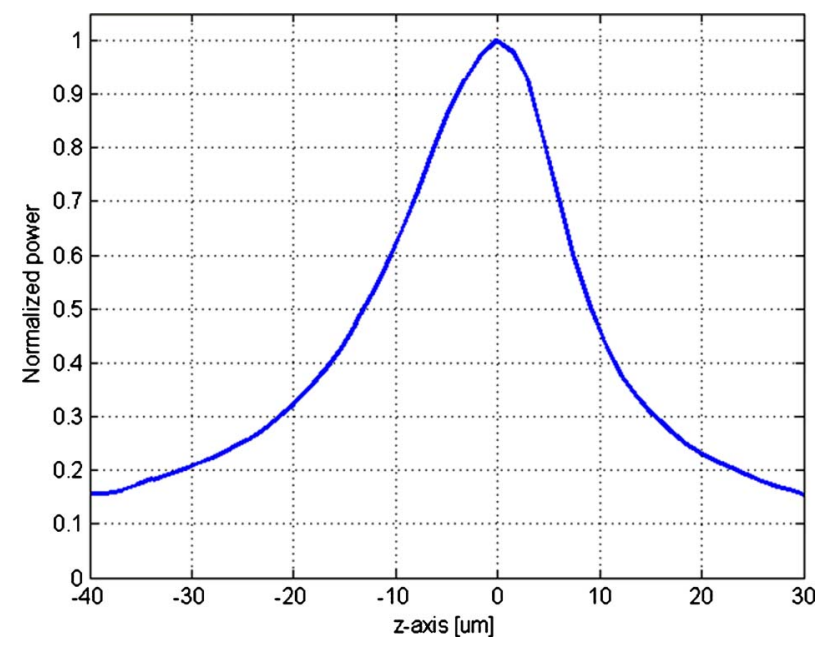

Fig. 6 Normalized diffraction efficiency versus $d z$ using 633-nm laser.

\section{Summary and Discussion}

We present the optical design and experimental data for an $S^{2}$-VHIS with improved field of view. Analysis of the data shows that the system has a resolution of approximately 22- $\mu \mathrm{m}$ axial (FWHM) and 4.5- $\mu \mathrm{m}$ lateral over a field of view of $1.2 \mathrm{~mm}$, which is an order of magnitude larger than previous systems. The axial and lateral resolution of this system can be further improved by increasing the angularspectral selectivity of the gratings. Theoretically, this can be achieved by increasing the thickness. However, in practice, a thicker grating does not necessarily have better selectivity if the light absorption of the holographic material is high. For our $S^{2}$-VHIS design, we estimate that with small modifications on our recording medium (PQ PMMA) concentration that an axial resolution of $\sim 10 \mu \mathrm{m}$ can be reached while for $\mathrm{SNR}>12 \mathrm{~dB}$.

This system simultaneously displays two depth sections separated by $\sim 70 \mu \mathrm{m}$, although other configurations with additional sections (five) have been demonstrated by our group. ${ }^{7}$ The number of depth section that a $\mathrm{S}^{2}$-VHIS can display depends on the number of holograms. However, the larger the number of holograms, the lower the SNR. At present our group is working on the optimization of five depth sections.

\section{Acknowledgments}

This work was supported in part by the National Institutes of Health (CA118167). Part of this work was performed while George Barbastathis was on sabbatical leave from MIT at the School of Engineering and Applied Science at Harvard University.

\section{References}

1. A. R. Tumlinson, L. P. Hariri, U. Utzinger, and J. K. Barton "Miniature endoscope for simultaneous optical coherence tomography and laser-induced fluorescence measurement," Appl. Opt. 43, 113-121 (2004).

2. A. R. Rouse and A. F. Gmitro, "Multispectral imaging with a confocal microendoscope," Opt. Lett. 25, 1708-1710 (2000).

3. B. K. Ford, M. R. Descour, and R. M. Lynch, "Large-image-format computed tomography imaging spectrometer for fluorescence microscopy," Opt. Express 9, 444-453 (2001).

4. J. G. Fujimoto and D. L. Farkas, Biomedical Optical Imaging, Oxford University Press, Cambridge, UK (2009).

5. W. Liu, G. Barbastathis, and D. Psaltis, "Volume holographic hyperspectral imaging," Appl. Opt. 43, 3581-3599 (2004).

6. A. Sinha, W. Sun, T. Shih, and G. Barbastathis, "Volume holographic imaging in transmission geometry," Appl. Opt. 43, 1533-1551 (2004).

7. Y. Luo, P. Gelsinger, J. K. Barton, G. Barbastathis, and R. K. Kostuk, "Optimization of multiplexed holographic gratings in PQ-PMMA for spectral-spatial imaging filters," Opt. Lett. 33, 566-568 (Mar. 2008).

8. H. Kogelnik, "Coupled wave theory for thick hologram gratings," Bell Syst. Tech. J. 48, 2909-2947 (1969)

9. M. G. Moharam and T. K. Gaylord, "Three-dimensional vector coupled-wave analysis of planar-grating diffraction," J. Opt. Soc. Am. 9, 1105-1112 (1983)

10. J. W. Goodman, Introduction to Fourier Optics, 2nd ed., McGrawHill, New York (1996)

11. G. Barbastathis, M. Balberg, and D. Brady, "Confocal microscopy with a volume holographic filter," Opt. Lett. 24(12), 811-813 (1999).

12. J. C. Wyant and K. Creath, "Basic wavefront aberration theory for optical metrology," in Applied Optics and Optical Engineering, R. R. Shanon and J. C. Wyant, Eds., Vol. XI, pp. 46-52, Harcourt, Brace, Jovanovich, New York (1992).

13. W. Smith, Modern Optical Engineering, 3rd ed., McGraw-Hill, New York (2000).

Biographies and photographs of the authors not available. 